\title{
BIOGEOCHEMISTRY OF BRACHIOPOD INTRACRYSTALLINE PROTEINS AND AMINO ACIDS.
}

WALTON*, Derek; CURRY, Gordon B., Department of Geology and Applied Geology, University of Glasgow, Lilybank Gardens, Glasgow, G12 8QQ, United Kingdom.

Brachiopods contain several classes of intracrystalline molecules, secreted during the growth of the organism, and subsequently incorporated in the shell during biomineralization. The function of these molecules is not clear, although in the Mollusca, molecules in similar sites are thought to be involved with biologically controlled mineralization. The order of amino acids, and hence the quantity of each amino acid in proteins is determined by the genome of the organism, and study of such molecules should reveal information regarding the genomes of the fossil organisms.

Previous studies of fossil molecules from brachiopods have concentrated on the web of intracrystalline molecules which surrounds the crystallites of the secondary fibres of the shell. Work with Recent articulate brachiopods has shown that this web decays in less than a year, and hence any remnants of this web in the fossil record are likely to.be highly degraded and unrepresentative of the original composition of the protein.

In contrast to this, intracrystalline molecules will be protected, encased within the calcium carbonate of the shell and analogous in many ways to fluid inclusions found in inorganic minerals. In such sites, contaminant and degradative factors such as microorganisms and large concentrations of water will be excluded and the effects of the enclosing sediments negated. Any breakdown of the molecules will be in situ, and the detectable products analysed.

In this study, intracrystalline molecules have been extracted from the shells of Recent and fossil brachiopods from the Plio-Pleistocene South Wanganui Basin, New Zealand. The molecules were analysed for free and peptide bound amino acids from both the soluble and insoluble fractions. The state of preservation of the molecules and their taxonomic specifity were analysed.

It was found that, even in these protected sites, individual amino acids were up to $90 \%$ in the free state i.e. that the proteins had mostly been hydrolyzed by the action of time and any water remaining in the shell structure after $0.12 \mathrm{Ma}$. Amino acids which are particularly sensitive to degradative reactions, such as serine and threonine were totally lost from the soluble fraction. However, the use of multivariate statistical analyses on data from the soluble remains of the protein indicates that decay does not render the molecules useless for taxonomy; groupings can be made on the basis of analyses alone.

Progressively older samples tend to have lower yields of amino acid in the soluble fraction, and this tends to correspond with an increase in the relative proportion of intracrystalline insoluble compounds. Although there is no direct linear relationship between the age of a sample and the proportion of insoluble compounds, older samples tend to have more insolubles. It is clear that other factors must play a part in the formation of these insoluble compounds. Preliminary studies of their amino acid composition indicates the presence of sensitive amino acids such as serine, indicating the stabilising effects of these diagenetic compounds.

The degradation of amino acids follows complex decay pathways, with some decaying to non-amino compounds and others to other amino acids, the most common being alanine from the dehydration of serine. The increasing concentrations of these amino acids can be a distorting factor in molecular taxonomy from fossil molecules.

ACKNOWLEDGEMENTS. This work was completed during the tenure of a UK NERC studentship to DW (GT4/89/GS/42) and funding from the Royal Society to GBC. 